\title{
Hermetic Housing Design for TR Module in LMDS System
}

\author{
Wen-Hua Tu, Chun-Long Wang, and Ruey-Beei Wu \\ Department of Electrical Engineering and Graduate Institute of Communication Engineering, \\ National Taiwan University, Taipei, Taiwan, 10617, ROC \\ E-mail: rbwu@ew.ee.ntu.edu.tw
}

\begin{abstract}
This paper presents a design of the hermetic housing for TR module in LMDS system by suppressing the cavity modes in closed channel. By suitably adding partition plates with aperture opening for signal line transmission, the cavity modes can be expelled off the operating frequency range. Three cases of no partition, four-partition, and six-partition are simulated and the performance are compared. An improvement in insertion loss of $20 \mathrm{~dB}$ was observed in measurement.
\end{abstract}

\section{Introduction}

In microwave and millimeter wave communication systems, RF front-end components, such as low noise amplifier, power amplifier, mixer, and oscillator are light, thin, and small. Since the components are vulnerable and friable during applications under the above trend, they are usually packaged into a robust and shielding cavity-like channel. Although the shielding channel prevents the RF components from external invasion, it may introduce unwanted cavity modes in the hermetic environment. When cavity modes occur at system's operation band, it may in the worst case cause system oscillation or instability if there happens to be a feedback path with proper feedback energy and phase delay. [1]

This paper describes how to suppress these cavity modes at operation band of system by adding additional partition plates. Mode patterns and resonant frequencies for channels with different number of partition plates will be simulated in this paper. Measured data demonstrate that coupling among RF components due to cavity modes can be effectively suppressed by adding suitable number of partition plates at suitable positions.

\section{Description of problem}

Consider a housing design for TR module in an LMDS system (Tx: 31.0-31.3GHz, Rx: 27.3528.35GHz). The transmitter and receiver channels are incorporated into a U-type channel (Fig. 1). The upper arm is for receiver and the lower one is for transmitter. The metals in the housing walls and the partition plates are assumed to be perfect conductor to facilitate the numerical simulation. A Teflon substrate $\left(\varepsilon_{\mathrm{r}}=3\right.$, thickness $\left.=0.254 \mathrm{~mm}\right)$ is placed above the bottom of the channel, elsewhere the space is filled with air.

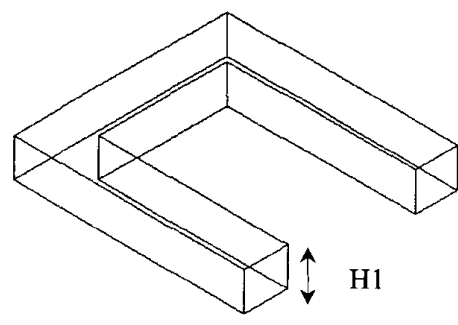

(a)

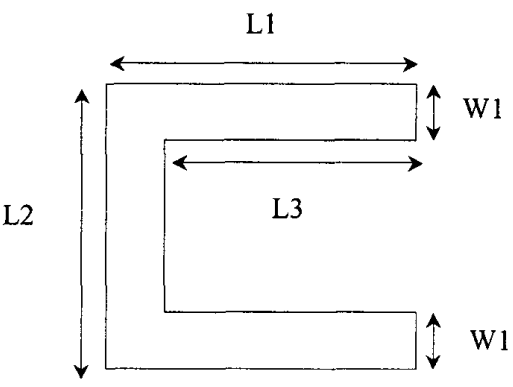

(b)

Fig. 1 Schematic of U-type channel (a) 3D view, (b) Top view $(\mathrm{H} 1=4.5 \mathrm{~mm}, \mathrm{~L} 1=27 \mathrm{~mm}, \mathrm{~L} 2=25 \mathrm{~mm}, \mathrm{~L} 3=22 \mathrm{~mm}$, $\mathrm{W} 1=\mathrm{W} 2=5 \mathrm{~mm}$ ) 


\section{Simulation and design}

Simulation is performed by the source-free Eigenmode solver of Ansoft/HFSS v. 7. To begin with, channel with substrate only is taken into consideration. Electric field distributions of the first four dominant modes are shown in Fig. 2. The resonant frequencies have been compared favorably with those by an in-house software based on 2D finite element waveguide solver together with transverse resonance concept. In order to expel the first mode with resonant frequency $28.26 \mathrm{GHz}$, some partition plates are added into the channel. Fig. 3 shows an example of four partition plates, over each of which an aperture opening with dimension $1.4 \mathrm{mmx} 2.4 \mathrm{~mm}$ is perforated to allow for signal line transmission. The positions of the partition plates are chosen not to intrude any individual major front-end component (such as LNA, PA and Filter). The simulation results are shown in Fig. 4. The electric filed distributions of the several lowest modes are similar to those of no-partition case, although the fields are confined in a smaller area. Comparing with no-partition case, the resonant frequencies do increase, but only in a slight amount. Two more partition plates are thus inserted along the central arm with carefully chosen positions. (Fig. 5) In Fig.6, it can be verified that there is no cavity mode in our operation band.
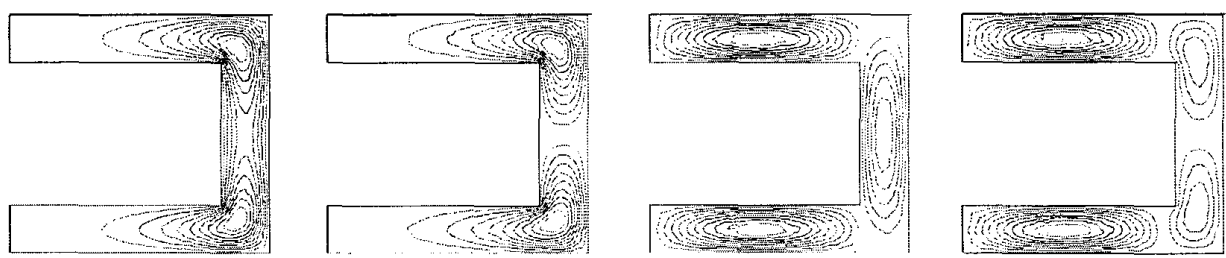

Fig. 2 Electric field distributions of the first four cavity modes with resonant frequencies of $28.26 \mathrm{GHz}$, $28.45 \mathrm{GHz}, 30.08 \mathrm{GHz}$, and $30.21 \mathrm{GHz}$, respectively.

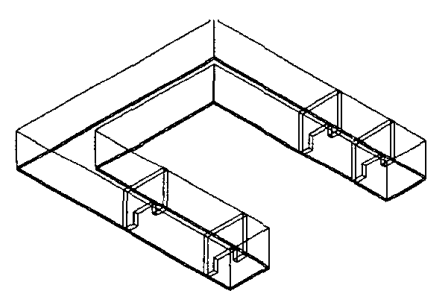

(a)
L4

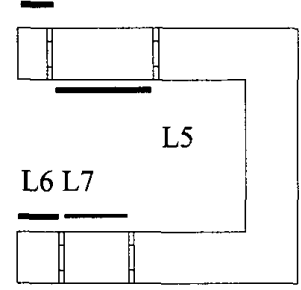

(b)

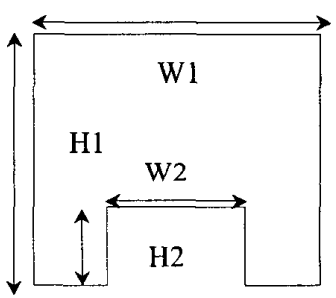

(c)

Fig. 3 Schematics of channel with four partition plates (a) 3D view, (b) top view, (c) cross sectional view of the partition plate. $(\mathrm{L} 4=2.8 \mathrm{~mm}, \mathrm{~L} 5=9.7 \mathrm{~mm}, \mathrm{~L} 6=4 \mathrm{~mm}, \mathrm{~L} 7=6.2 \mathrm{~mm}, \mathrm{H} 2=1.4 \mathrm{~mm}, \mathrm{~W} 2=2.4 \mathrm{~mm}$, board thickness $=0.5 \mathrm{~mm}$ )
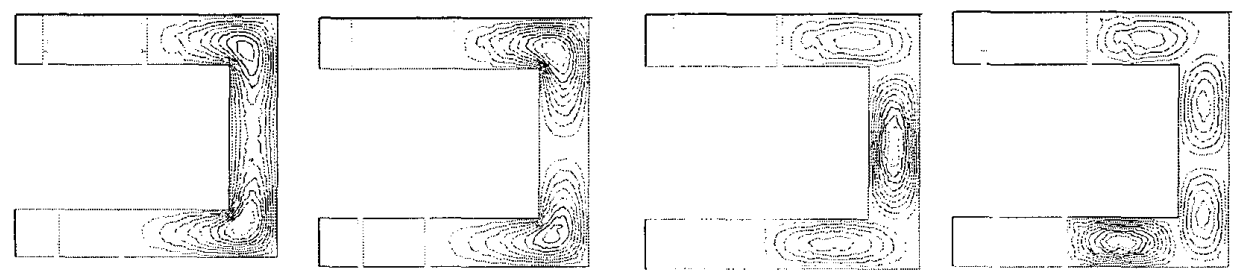

Fig. 4 Electric field distribution of the first four cavity modes with resonant frequency of $28.30 \mathrm{GHz}, 28.53 \mathrm{GHz}$, $30.91 \mathrm{GHz}$, and $32.35 \mathrm{GHz}$, respectively. 


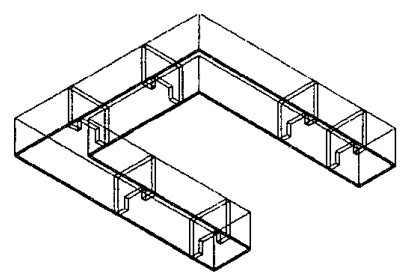

(a)

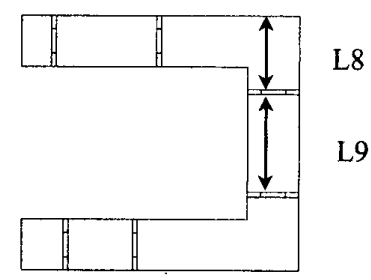

(b)

Fig. 5 Schematics of channel with six partition plates (a) $3 \mathrm{D}$ view, (b) top view, (L8=7.2mm, L9 $=10 \mathrm{~mm}$ )
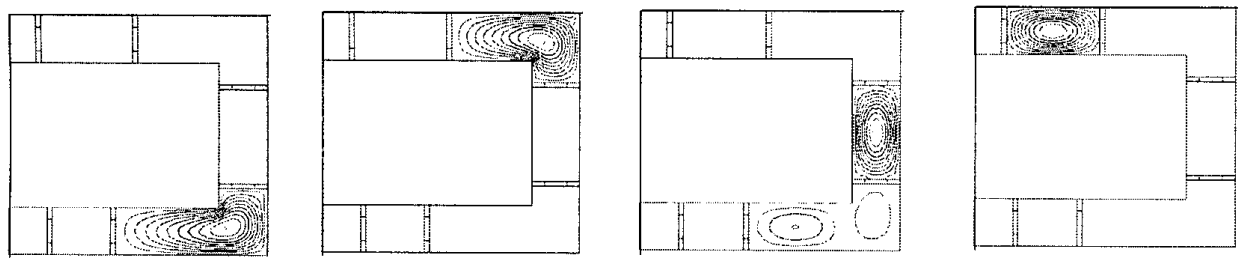

Fig. 6 Electric field distribution of the first four cavity modes with resonant frequency of $29.36 \mathrm{GHz}, 29.39 \mathrm{GHz}$, $32.68 \mathrm{GHz}$, and $32.76 \mathrm{GHz}$, respectively.

\section{Experiment and measurement}

Schematic and photograph of test sample are shown in Fig. 7. In order to excite cavity modes, two open-ended $50 \mathrm{ohm}$ microstrip lines connected to $\mathrm{K}$ connectors were employed. The partition plates are soldered to channel, and can be removed to investigate the effects of each plate. A lid (shown as the right side in Fig. 7(a)) with nine holes is screwed up to guarantee a closed cavity. The measured insertion loss of four-partition and six-partition channels is shown in Fig. 8. Insertion loss of six-partition channel is around $-50 \mathrm{~dB}$, as opposed to a maximum insertion loss of $-30 \mathrm{~dB}$ for fourpartition channel. Significant improvement of insertion loss, especially for the band $30-32 \mathrm{GHz}$, has been achieved by adding two additional plates, which has freed off all cavity modes in the operating frequency range. Although, prominent peaks in insertion loss as expected from the cavity modes did not appear in measurement, which may be due to negligence of substrate and metal loss, and leads to a smaller quality factor (i.e. broader bandwidth).

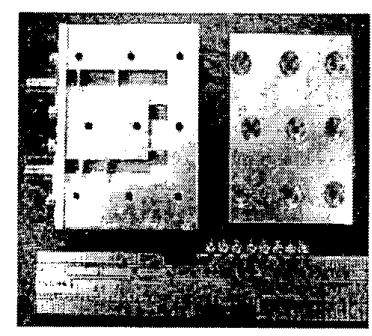

(a)

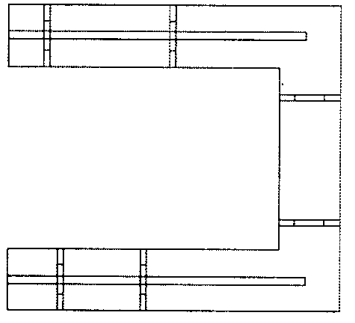

(b)

Fig. 7 Photograph and schematic of test sample. (a) Photograph of sample, (b) Schematic 


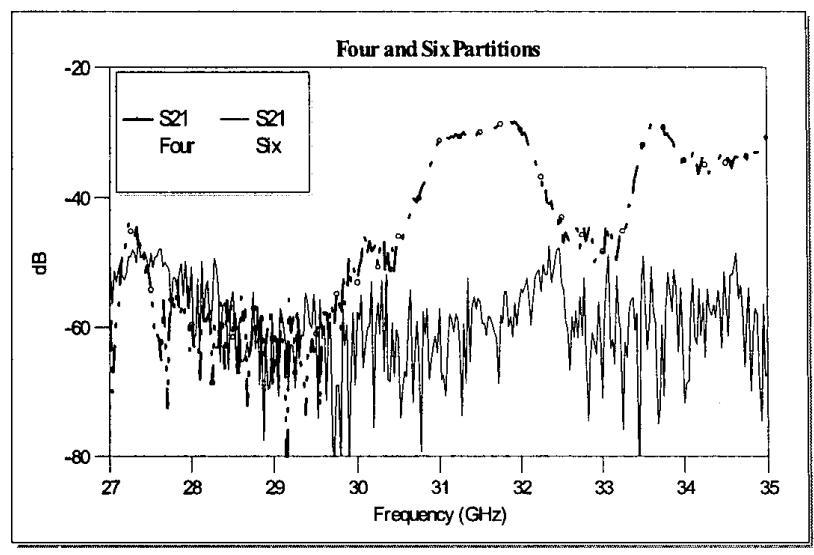

Fig. 8 Measured insertion loss of channel with four and six plates (Dot line: four-board channel, solid line: sixboard channel)

\section{Conclusions}

In order to suppress cavity modes in closed channel, partition plates with aperture opening are employed. With suitable design, the cavity modes can be expelled off the operation band of system. In six-partition channel, no cavity mode occurs in operation band and $20 \mathrm{~dB}$ improvement in insertion loss has been demonstrated by measurement.

\section{Acknowledgement}

The authors would like to acknowledge Jay-Chao Liu in Airwave Corp. for his help in fabrication and assembly. Thanks also to National Center for High-performance Computing (NCHC) for providing the simulation environment of Ansoft/HFSS v.7. This work was supported in part under Grant NSC89-2213-E002-051 by National Science Council, Grant 89-E-FA06-2-4 by Ministry of Education, and Grant 88-S-052 by AirWave Technology Inc. Republic of China.

\section{References}

[1] G. Gonzales, Microwave Transistor Amplifiers Analysis and Design, Chapter 5, $2^{\text {nd }}$ ed., Prentice-Hall, N. J., 1997. 